\title{
Evaluation of choroidal thickness measurements in pediatric obstructive sleep apnea syndrome patients
}

\author{
Cem Bayraktar ${ }^{1}$, Ali Şimşek ${ }^{2}$ \\ ${ }^{1}$ Department of Otolaryngology, Head and Neck Surgery, ${ }^{2}$ Department of Ophthalmology, Adryaman University Research \\ and Training Hospital, Adıyaman, Turkey.E-mail:cem_dr23@hotmail.com \\ Received: 21st December 2016, Accepted: 28th March 2017
}

SUMMARY: Bayraktar C, Şimşek A. Evaluation of choroidal thickness measurements in pediatric obstructive sleep apnea syndrome patients. Turk J Pediatr 2017; 59: 62-67.

Choroidal vascular impairment and obstructive sleep apnea syndrome (OSAS) related disorders have been shown in adults, but there have been no investigations with regard to the choroidal thickness measurements in children with OSAS. Our aim was to investigate this relationship in the early childhood period, which correlates with OSAS peak incidence due to adenotonsillar enlargement. One-hundred and nine pediatric OSAS patients and 42 age-sex matched controls were enrolled in this study. Nocturnal pulse oximetry was used to define the OSAS patients. Demographic data like age, sex and body-mass index were recorded. All of the subjects underwent complete otolaryngologic and ophthalmologic examination including visual acuity, intraocular pressure, central corneal thickness, axial length and choroidal thickness measurements. Overall, the $1,000 \mu \mathrm{m}$ and 1,500 $\mu \mathrm{m}$ nasal choroid measurements were found to be significantly lower in the patient group $(\mathrm{p}<0.05)$. However, only a poor inverse correlation emerged between the body-mass index (BMI) and the foveal center measurements in the patients. Observed alterations in the choroid may signify the initial impairment of known OSAS-related eye disorders, like glaucoma, floppy eyelid syndrome, and non-arteritic ischemic optic neuropathy. Ophthalmologists, otolaryngologists and pediatricians working together as a team may be able to prevent future eye diseases.

Key words: adenotonsillar hypertrophy, choroid, nocturnal pulse oximetry, sleep apnea.

Adenotonsillar hypertrophy is the most common cause of obstructive sleep apnea syndrome (OSAS) in the pediatric age group, and an adenotonsillectomy is the first line of treatment in those patients ${ }^{1,2}$. OSAS is common, affects 1 to 3 percent of children in childhood ${ }^{3}$, and is characterized by recurrent upper airway obstruction (partially or completely) during sleep ${ }^{4}$. The gold standard diagnosis method is full-night polysomnography (PSG), but it is time-consuming and expensive ${ }^{5}$. Additionally, sleep laboratories have little experience in pediatric testing. Although the medical history and physical examination are very important, in circumstances when polysomnography is unavailable, other objective testing is recommended, including nocturnal video recording, nocturnal oximetry, and ambulatory polysomnography ${ }^{6}$. Recently, nocturnal oximetry was defined as an easy to use and low-cost diagnostic test when polysomnography is not available ${ }^{7}$.

The choroid is a vascular layer which lies between the sclera and the retina ${ }^{8}$. It plays important roles in the physiology of the eye, such as supplying oxygen and nutrients to the outer retina, maintaining intraocular pressure, regulating ocular temperature, absorbing light, and removing waste products 9,10 . The choroid is one of the most highly vascularized tissues in humans, and due to OSAS, hypoxia, hypercapnia, impaired balance in the endothelial vasoactive substances, hemodynamic changes, and autonomic dysfunction may all cause impairment in the choroidal blood flow ${ }^{11}$. In previous studies, alterations in the choroidal 
thickness have been shown in adults with OSAS $^{1-14}$. To the best of our knowledge, this is the first study to investigate the choroidal blood flow in the pediatric OSAS population. Our aim was to investigate the alterations in the choroidal thickness in children with adenotonsillar hypertrophy.

\section{Material and Methods}

This study was performed in the Otolaryngology and Ophthalmology Departments. Ethical Committee approval was obtained from our tertiary hospital, and the study was executed while abiding by the Declaration of Helsinki. Written informed consent was obtained from all subjects.

One-hundred and nine children, aged 3 to 8 years old (early childhood period), with adenotonsillar hypertrophy and OSAS, and 42 age and sex matched controls were evaluated in this study. The demographic characteristics of the patients, such as the age, sex, and body-mass index (BMI), were recorded. All of the patients underwent detailed otolaryngological and ophthalmological examinations, including nasal endoscopy and optical coherence tomography (OCT). The palatine tonsils were graded as follows: Grade 1 - in palatine fossa, Grade 2 - can be observed behind anterior pillar, Grade 3 - medial to the pillars without obstruction, and Grade 4 - causing airway obstruction. The size of the adenoids was graded into 3 categories as described by Wang et al. ${ }^{15}$. The distance between the vomer and anterior border of the adenoid tissue was assessed using flexible endoscopy, with $>1 \mathrm{~cm}$ defined as "small", 0.5-1 cm defined as "moderate," and smaller than $0.5 \mathrm{~cm}$ defined as "large". Those patients included in the study were between 3 and 8 years old, with Grades 3-4 tonsillar and moderate to large adenoidal hypertrophy, and open mouth sleeping, snoring, and witnessed apnea. Nocturnal pulse oximetry was performed on all of the children, including the controls, and desaturation was defined as a $4 \%$ or more $\mathrm{SaO}_{2}$ decrement, and desaturation cluster was defined as 5 or more desaturation in a 10 to 30 minute period ${ }^{16}$. All of the subjects and controls underwent at least six-hour assessments with nocturnal pulse oximetry, and those patients with 3 or more clusters of desaturation, and a minimum of 3 desaturations below $90 \%$, were defined as having OSAS7.
Those subjects with known eye disease, chronic illness (diabetes, cardiovascular disease, etc.) diabetic retinopathy, a history of intraocular surgery, ocular trauma, peripapillary choroidal atrophy, refractive errors, medical treatment for any reason, or any vascular disease were excluded from this study.

\section{Choroidal thickness and other eye parameter measurements}

The following ophthalmological examinations were performed in each of the subjects: slit-lamp, dilated fundus, refractive error, visual acuity, and intraocular pressure (IOP) measurements. The best visual acuity values were measured using a Snellen chart, and the axial length of the eye was measured with an optical biometer (LENSTAR LS 900; Haag-Streit AG, Koeniz, Switzerland). A spectral domain OCT system (RTVue-100 OCT; Optovue Inc., Fremont, CA, USA), using software version 6.3 , was used to obtain the choroidal images with undilated pupils. Two experienced ophthalmologists recorded the measurements separately, and the values were averaged. Images with signal strength indexes (SSIs) >60 were included in this study. Diurnal variations may exist in the choroidal thickness measurements ${ }^{17}$; therefore, all of the measurements were performed between 13:00 and 15:00 (1 and 3 pm), and the right eye measurements were used for the statistical analyses. The choroid was measured at seven points: the foveal center, and between the horizontal nasal and temporal quadrants at 500 micron intervals, to a distance of 1,500 microns in both directions. An optional attached lens $(\mathrm{CAM}-\mathrm{L})(8 \times 1024 \mathrm{~A}$-scans $)$ was used for the central corneal thickness (CCT) measurements. The CCT was measured using a pachymetry map of the OCT, and images with SSIs $>30$ were included.

\section{Statistical analysis}

SPSS 15.0 for Windows (SPSS Inc., Chicago, USA) was used for the statistical analysis, and the categorical variables were shown as percentages and analyzed using Fisher's exact test. The data normality was tested with the Kolmogorov-Smirnov test. Because the data distribution was not normal, the MannWhitney $U$ test was used to compare the data between the groups. The Pearson correlation coefficient was used to assess the relationship between the foveal center measurements and 
Table I. Demographic and Ocular Characteristics of the Groups

\begin{tabular}{llll}
\hline Characteristics & Control group $(\mathrm{n}=42)$ & Patient group $(\mathrm{n}=109)$ & P value $^{*}$ \\
\hline Age (years) & $5.23 \pm 0.75$ & $5.39 \pm 0.90$ & 0.453 \\
Female (\%) & 45 & 49 & $0.717^{* *}$ \\
BMI $\left(\mathrm{kg} / \mathrm{m}^{2}\right)$ & $16.21 \pm 1.70$ & $15.93 \pm 1.32$ & 0.455 \\
\hline
\end{tabular}

*Mann Whitney-U test; ** Fisher's exact test; BMI: body-mass index

the other demographic/ocular characteristics. The descriptive data was shown as the mean \pm standard deviation.

\section{Results}

One-hundred and nine patients with adenotonsillar hypertrophy and 42 age and sex matched controls were enrolled in this prospective study. The average age was 5.39 \pm 0.90 years old in the patient group and $5.23 \pm 0.75$ years old in the control group. The age, sex, and BMI were not observed to be statistically different between the groups ( $>0.05$, Table I).

There was a significant difference in the choroidal thickness measurements with regard to the $1,000 \mu \mathrm{m}$ and $1,500 \mu \mathrm{m}$ nasal measurements $(p<0.05)$, but the other choroidal parameters and eye measurements, including the IOP, CCT, visual acuity, and axial length, were not significantly different between the groups $(p>0.05$, Table II).

The correlation analysis revealed that there was only a poor inverse relationship between the $\mathrm{BMI}$ and the foveal center measurements in the patients $(\mathrm{r}=-0.251, \mathrm{p} \leq 0.001$, Table III $)$

\section{Discussion}

The core findings of this study were: i) significant choroidal thickness thinning was observed in the nasal quadrant of the retina in children with OSAS; ii) there was no significant difference in the IOP, CCT, visual acuity, and axial length parameters in the OSAS patients when compared with the controls; and iii) there was no significant correlation between the foveal center thickness and the age, CCT, IOP, visual acuity, or axial length parameters, with the exception of a poor inverse correlation with the BMI.

OSAS is a common disease in childhood, and may result in complications if it is not properly treated. The risk factors include obesity, adenotonsillar hypertrophy, neuromuscular disorders, and craniofacial anomalies ${ }^{6}$. The incidence of OSAS is elevated during the early childhood period (3 to 8 years old), and at the same time, adenotonsillar tissue enlargement occurs during this period ${ }^{18}$. Snoring is the most common symptom in

Table II. Comparisons of Choroidal Thicknesses in the Study and Control Groups

\begin{tabular}{llll}
\hline & Control group $(\mathrm{n}=42)$ & Patient group $(\mathrm{n}=109)$ & P value* \\
\hline Foveal center $\mu \mathrm{m}$ & $317.21 \pm 26.20$ & $320.22 \pm 33.68$ & 0.785 \\
Temporal $500 \mu \mathrm{m}$ & $303.26 \pm 26.35$ & $307.24 \pm 33.41$ & 0.664 \\
Temporal $1,000 \mu \mathrm{m}$ & $293.38 \pm 27.87$ & $297.85 \pm 32.04$ & 0.702 \\
Temporal $1,500 \mu \mathrm{m}$ & $286.92 \pm 30.77$ & $289.95 \pm 32.67$ & 0.858 \\
Nasal $500 \mu \mathrm{m}$ & $300.76 \pm 25.29$ & $302.97 \pm 33.44$ & 0.847 \\
Nasal $1,000 \mu \mathrm{m}$ & $284.28 \pm 27.64$ & $266.76 \pm 35.83$ & 0.003 \\
Nasal $1,500 \mu \mathrm{m}$ & $274.09 \pm 30.47$ & $251.13 \pm 36.02$ & 0.001 \\
IOP $(\mathrm{mmHg})$ & $13.73 \pm 1.10$ & $13.95 \pm 1.21$ & 0.337 \\
CCT $(\mu \mathrm{m})$ & $540.76 \pm 17.30$ & $543.43 \pm 22.36$ & 0.545 \\
Visual acuity $($ Snellen$)$ & $1.20 \pm 0.10$ & $1.18 \pm 0.08$ & 0.116 \\
Axial length $(\mathrm{mm})$ & $22.04 \pm 0.76$ & $21.91 \pm 0.80$ & 0.358 \\
\hline
\end{tabular}

* Mann Whitney-U test; CCT: central corneal thickness; IOP: intraocular pressure 
OSAS patients, with the additional symptoms consisting of irritability, nocturnal enuresis, hyperactivity, night gasping and sweats, and behavioral problems. This process may result in systemic arterial hypertension, pulmonary vascular enhancement, or cor pulmonale in some cases ${ }^{19,20}$. Increased sympathetic system, endothelial, and autonomic dysfunctions have been shown in OSAS patients, and choroidal microcirculation, which is maintained by autonomic regulation, may be impaired as a result of one or more of these altered mechanisms ${ }^{11,13}$.

Choroidal blood flow alterations in adult OSAS patients have been an issue of some controversy in the literature. For example, Karaca et al. ${ }^{14}$ investigated the choroidal blood flow in 74 adult OSAS patients, and reported that there was no significant difference in the choroidal thickness between the OSAS groups (separated into 3 subgroups: mild, moderate, and severe) and the controls. They reported that all of the OSAS patients were newly diagnosed, and that the cause of the lack of deterioration in the choroidal flow may have been still intact autoregulatory mechanisms. Tonini et al. ${ }^{13}$ found similar choroidal blood flow reactivity to $\mathrm{CO}_{2}$ and $\mathrm{O}_{2}$ in the OSAS patients and controls in their prospective study, and concluded that ocular microcirculation, which provides adaptive mechanisms, continues long-term in OSAS patients. Contrarily, Xin et al. ${ }^{12}$ observed significantly lower measurements in severe OSAS patients with regard to the subfoveal and nasal choroidal thicknesses, when compared with the mild-moderate OSAS patients and control subjects. Additionally, Bayhan et al. ${ }^{11}$ reported significantly thinner choroidal thicknesses $1.5 \mathrm{~mm}$ and $3.0 \mathrm{~mm}$ nasal to the fovea in severe OSAS patients, when compared with the controls. In this study, we observed significantly lower $1,000 \mu \mathrm{m}$ and 1,500 $\mu \mathrm{m}$ nasal choroidal thickness measurements when compared with the controls.

The normal function and structure of the choroid is essential for proper retinal function $11,12,14$. The choroid mainly supplies the outer layers of the retina, which contain photoreceptors, and abnormal flow changes may cause photoreceptor death or dysfunction ${ }^{21}$. In the healthy subjects, the subfovea was observed to be the thickest portion, and the choroidal thickness to the periphery was reduced gradually, especially on the nasal side $^{12}$. Our findings were in accordance with the literature, and we found thinner average measurements in the nasal part of the macula in both the controls and the patients.

The choroidal thickness measurements may be affected by factors like the perfusion pressure, axial length, CCT, and age ${ }^{22}$. Therefore, we performed ocular characteristic measurements in both groups to obtain the true choroidal thickness measurements. In addition, we observed an inverse relationship between the BMI and foveal center measurements in the patients, and believe that this relationship makes sense, because obesity is one of the factors contributing to OSAS.

The strengths of this study were, first, we used the SD-OCT for the choroidal measurements, which is a reproducible, non-invasive, effective, and easy tool for measuring 23 . Second, this was the first study to evaluate the choroidal thickness measurements in the pediatric OSAS population. However, there were several limitations to this research; for example, the gold standard method for the diagnosis of OSAS is polysomnography, but it is not

Table III. Correlation Between Demographic/ Ocular Characteristics of Patients with OSAS and the Choroidal Thickness of the Foveal Center

\begin{tabular}{lll}
\hline Characteristics & $\mathrm{r}$ & $\mathrm{p}^{*}$ \\
\hline Age (year) & 0.045 & 0.645 \\
BMI $\left(\mathrm{kg} / \mathrm{m}^{2}\right)$ & -0.251 & $<0.001$ \\
CCT $(\mu \mathrm{m})$ & 0.080 & 0.411 \\
IOP $(\mathrm{mmHg})$ & 0.022 & 0.817 \\
Visual acuity (Snellen) & 0.125 & 0.194 \\
Axial length (mm) & -0.044 & 0.647 \\
\hline
\end{tabular}

*Pearson correlation; BMI: body-mass index; CCT: central corneal thickness; IOP: intraocular pressure 
available in our institute. In addition, we did not know the duration of the OSAS that the patients were exposed to. Moreover, it was not determined whether an adenotonsillectomy, which is the first line therapy in children with OSAS, causes a return to the choroidal pathology. An evaluation of these factors may provide additional information about choroidal thickness changes in pediatric OSAS patients, and should be addressed in future studies.

Ophthalmic findings, like floppy eyelid syndrome, nonarteritic ischemic optical neuropathy, and glaucoma may be associated with OSAS, and these findings may help clinicians to identify undiagnosed OSAS patients ${ }^{24}$. The exact mechanism between OSAS and these disorders has not yet been clarified ${ }^{11}$. However, we suggest that the impairment of the choroidal thickness could be one of the underlying pathophysiological processes in these patients. Children who suffer from OSAS due to adenotonsillar hypertrophy may benefit from referrals to an ophthalmologist and otolaryngologist, thereby preventing more serious eye diseases.

In conclusion, we observed significantly thinner choroidal thicknesses in the $1,000 \mu \mathrm{m}$ and $1,500 \mu \mathrm{m}$ nasal choroidal measurements in patients with OSAS due to adenotonsillar hypertrophy. A diagnosis of OSAS should be kept in mind in children who have choroidal alterations. Otolaryngologists working with ophthalmologists and pediatricians, together as a team, may enhance the awareness of the relationship between these diseases, and could prevent more severe eye pathologies from occurring.

\section{REFERENCES}

1. Ray RM, Bower CM. Pediatric obstructive sleep apnea: the year in review. Curr Opin Otolaryngol Head Neck Surg 2005; 13: 360-365.

2. Shine NP, Lannigan FJ, Coates HL, Wilson A. Adenotonsillectomy for obstructive sleep apnea in obese children: effects on respiratory parameters and clinical outcome. Arch Otolaryngol Head Neck Surg 2006; 132: 1123-1127.

3. Nixon GM, Kermack AS, Davis GM, Manoukian JJ, Brown KA, Brouillette RT. Planning adenotonsillectomy in children with obstructive sleep apnea: the role of overnight oximetry. Pediatrics 2004; 113: e19-25.
4. Statham MM, Elluru RG, Buncher R, Kalra M. Adenotonsillectomy for obstructive sleep apnea syndrome in young children: prevalence of pulmonary complications. Arch Otolaryngol Head Neck Surg 2006; 132: 476-480.

5. Baldassari CM, Mitchell RB, Schubert C, Rudnick EF. Pediatric obstructive sleep apnea and quality of life: a meta-analysis. Otolaryngol Head Neck Surg 2008; 138: 265-273.

6. Marcus CL, Brooks LJ, Draper KA, et al. Diagnosis and management of childhood obstructive sleep apnea syndrome. Pediatrics 2012; 130: e714-755.

7. Kaditis A, Kheirandish-Gozal L, Gozal D. Pediatric OSAS: Oximetry can provide answers when polysomnography is not available. Sleep Med Rev 2016; 27: 96-105.

8. Shao Z, Dorfman AL, Seshadri S, et al. Choroidal involution is a key component of oxygen-induced retinopathy. Invest Ophthalmol Vis Sci 2011; 52: 6238-6248.

9. Read SA, Collins MJ, Vincent SJ, Alonso-Caneiro D. Choroidal thickness in childhood. Invest Ophthalmol Vis Sci 2013; 54: 3586-3593.

10. Parver LM. Temperature modulating action of choroidal blood flow. Eye (Lond) 1991; 5: 181-185.

11. Bayhan HA, Aslan Bayhan S, İntepe YS, Muhafiz E, Gürdal C. Evaluation of the macular choroidal thickness using spectral optical coherence tomography in patients with obstructive sleep apnoea syndrome. Clin Experiment Ophthalmol 2015; 43: 139-144.

12. Xin C, Wang J, Zhang W, Wang L, Peng X. Retinal and choroidal thickness evaluation by SD-OCT in adults with obstructive sleep apnea-hypopnea syndrome (OSAS). Eye (Lond) 2014; 28: 415-421.

13. Tonini M, Khayi H, Pepin JL, et al. Choroidal bloodflow responses to hyperoxia and hypercapnia in men with obstructive sleep apnea. Sleep 2010; 33: 811-818.

14. Karaca EE, Ekici F, Yalçın NG, Çiftçi TU, Özdek Ş. Macular choroidal thickness measurements in patients with obstructive sleep apnea syndrome. Sleep Breath 2015; 19: 335-341.

15. Wang DY, Bernheim N, Kaufman L, Clement P. Assessment of adenoid size in children by fibreoptic examination. Clin Otolaryngol Allied Sci 1997; 22 172-177.

16. Brouillette RT, Morielli A, Leimanis A, Waters KA, Luciano R, Ducharme FM. Nocturnal pulse oximetry as an abbreviated testing modality for pediatric obstructive sleep apnea. Pediatrics 2000; 105: 405-412.

17. Tan CS, Ouyang Y, Ruiz H, Sadda SR. Diurnal variation of choroidal thickness in normal, healthy subjects measured by spectral domain optical coherence tomography. Invest Ophthalmol Vis Sci 2012; 53 261-266.

18. Brietzke SE, Gallagher D. The effectiveness of tonsillectomy and adenoidectomy in the treatment of pediatric obstructive sleep apnea/hypopnea syndrome: a meta-analysis. Otolaryngol Head Neck Surg 2006; 134: 979-984. 
19. Valera FC, Avelino MA, Pettermann MB, et al. OSAS in children: correlation between endoscopic and polysomnographic findings. Otolaryngol Head Neck Surg 2005; 132: 268-272.

20. Tatlipinar A, Duman D, Uslu C, Egeli E. The effects of obstructive sleep apnea syndrome due to adenotonsillar hypertrophy on the cardiovascular system in children. Turk J Pediatr 2011; 53: 359-363.

21. Shiragami C, Shiraga F, Matsuo T, Tsuchida Y, Ohtsuki H. Risk factors for diabetic choroidopathy in patients with diabetic retinopathy. Graefes Arch Clin Exp Ophthalmol 2002; 240: 436-442.
22. Vural AD, Kara N, Sayin N, Pirhan D, Ersan HB Choroidal thickness changes after a single administration of coffee in healthy subjects. Retina 2014; 34: 1223 1228.

23. Branchini L, Regatieri CV, Flores-Moreno I, Baumann B, Fujimoto G, Duker JS. Reproducibility of choroidal thickness measurements in across three spectral domain optical coherence tomography systems. Ophthalmology 2012; 119: 119-123.

24. Abdal H, Pizzimenti JJ, Purvis CC. The eye in sleep apnea syndrome. Sleep Med 2006; 7: 107-115. 